\title{
Importance of neonatal screening for primary immunodeficiencies
}

Jung Woo Rhim, MD, PhD

Departments of Pediatrics, Daejeon St. Mary's Hospital, College of Medicine, The Catholic University of Korea, Daejeon, Korea

\begin{abstract}
Key message
- Early detection of asymptomatic infants with primary immunodeficiencies before the onset of infections enables effective treatment and intervention to prevent serious sequelae.

- T-cell receptor excision circles and kappa-deleting recombination excision circles have recently been used to detect $\mathrm{T}-$ or B-cell lymphopenia in neonates.
\end{abstract}

Primary immunodeficiencies (PIDs) are a heterogeneous group of inborn errors of immunity including $\mathrm{T}$ - and B-cell deficiencies, complement deficiencies, phagocyte disorders, and combined immunodeficiencies. With the novel progress in immunological and molecular techniques, including the development of diagnostic genetic tests, 430 PID have been identified and characterized. ${ }^{1)}$ PID predispose individuals to recurrent or unusual infections and are associated with other immune disorders including autoimmune diseases and lymphomas. ${ }^{2)}$ It is difficult to identify infants with PID prior to the onset of clinical symptoms. Thus, some patients may remain untreated for several years, which leads to many complications. Neonatal screening for PID enables the early identification of asymptomatic infants with a PID. Early diagnosis and intervention prevent serious sequelae.

Assays for T-cell receptor excision circles (TRECs) and kappa-deleting recombination excision circles (KRECs) are being increasingly used to screen for PID in many countries worldwide. For example, TREC-based PID screening programs were established in the majority of American states, Israel, Taiwan, Qatar, and Canada. The Netherlands, Japan, France, Spain, Norway, France, the United Kingdom, Turkey, Saudi Arabia, Iran, Iceland, Denmark, and Brazil have applications in progress to commence screening programs. ${ }^{3)}$ In Iran, between 2014 and 2016, neonatal screening for PID was performed of 2,160 newborns and identified 51 cases of severe combined immunodeficiency (SCID), agammaglobulinemia, and ataxia telangiectasia. ${ }^{4}$

TREC are small circular pieces of episomal DNA that are formed during T-cell receptor rearrangement within the thymus. TREC is an accurate measure of thymic function because it arises in the late phase of thymocyte maturation. Approximately $70 \%$ of T cells generate TREC. Several properties were established for TREC that make them useful markers of thymic output: high stability, not prone to degradation, and not replicated when cells divide. ${ }^{5)}$ The development of a TREC assay utilizing dried blood spots enabled neonatal screening for SCID as a public health measure. SCID are caused by diverse genetic mutations that lead to the absence of all T-cell functions and in a lack of $\mathrm{B}$ cells and natural killer cells. SCID is the most severe PID. Affected patients present with chronic diarrhea, pneumonia, sepsis, and failure to thrive. Recurrent infections with opportunistic organisms including Pneumocystis jiroveci, Candida albicans, adenovirus, cytomegalovirus, and varicella zoster virus. The early detection of SCID should be considered a pediatric emergency because making a diagnosis prior to the onset of severe infections permits lifesaving hematopoietic stem cell transplantation (HSCT). Recent evidence suggests that patient outcomes are markedly improved if HSCT is performed before the age of 3.5 months. ${ }^{6}$

The TREC assay can also be used to identify syndromes with T-cell impairments, such as Down syndrome (trisomy 21), Edwards syndrome (trisomy 18), and ataxia telangiectasia. ${ }^{7}$ Like $\mathrm{T}$ cells, in the process of $\mathrm{B}$-cell maturation, KREC is produced by kappa-deletion recombination events that determine allelic and isotypic exclusion of the gamma chain. ${ }^{8)} \mathrm{B}$-cell maturation defects in agammaglobulinemia occur before kappa-deleting recombination, KREC should not be produced. Therefore, KREC measurements are used to identify cases of asymptomatic agammaglobulinemia, which comprise around $30 \%$ of all antibody deficiencies. Agammaglobulinemia patients present with B-cell differentiation arrest in the bone marrow and the consequent absence of mature B cells and profound deficiency of all immunoglobulins (Ig). In these patients, recurrent infections appear after 6 months of age, whereas the mean age at diagnosis is 3 years. ${ }^{9)}$ This diagnostic delay results in frequent infections such as pneumonia, sepsis, meningitis, and other bacterial infections. Live viral vaccines such as those for measles, mumps, and rubella and chicken pox may be lethal to these children. Thus, early diagnosis and treatment including periodic Ig replacement therapy are essential to improving patient

Corresponding author: Jung Woo Rhim, MD, PhD. Department of Pediatrics, Daejeon St. Mary's Hospital, College of Medicine, The Catholic University of Korea, 64 Daeheung-ro, Jung-gu, Daejeon 34943, Korea 
Table 1. Cellular and antibody deficiencies detectable by TREC and KREC screening

\begin{tabular}{ll}
\hline Low TREC & Severe combined immunodeficiency \\
& DiGeorge syndrome (22q11.2 deletion syndrome) \\
& Combined immunodeficiency \\
& Down syndrome (trisomy 21) \\
& Edwards syndrome (trisomy 18) \\
& Ataxia telangiectasia \\
Low KREC & X-linked agammaglobulinemia \\
& AR-agammaglobulinemia \\
& Severe combined Immunodeficiency (T-B-) \\
\hline
\end{tabular}

KREC, kappa-deleting recombination excision circle; TREC, T-cell receptor excision circle; AR, autosomal recessive.

prognosis. ${ }^{10)}$ TREC/KREC-based screening enables the early identification of asymptomatic infants with cellular and antibody deficiencies (Table 1) and facilitating diagnostic precision, appropriate treatment, and improved clinical outcomes.

\section{Footnotes}

Conflict of interest : No potential conflicts of interest relevant to this article are reported.

See the article "Modern diagnostic capabilities of neonatal screening for primary immunodeficiencies in newborns" via https://doi.org/10.3345/cep.2020.01270.

\section{References}

1. Bousfiha A, Jeddane L, Picard C, Al-Herz W, Ailal F, Chatila T, et al. Human inborn errors of immunity: 2019 update on the classification from the International Union of Immunological Societies Expert Committee. J Clin Immunol 2020;40:66-81.

2. Primary immunodeficiency diseases: report of a WHO scientific group. Clin Exp Immunol 1995;99:1-24.

3. King J, Ludvigsson J, Hammarström L. Newborn screening for primary immunodeficiency diseases: the past, the present and the future. Int J Neonatal Screen 2017;3:19.

4. Nourizadeh M, Shakerian L, Borte S, Fazlollahi M, Badalzadeh M, Houshmand M, et al. Newborn screening using the TREC/KREC assay for severe T and B cell lymphopenia in Iran. Scand J Immunol 2018 Jun 26:e12699. https://doi.org/10.1111/sji.12699. [Epub].

5. Arellano MV, Ordonez A, Ruiz-Mateos E, Leal-Noval SR, Molina-Pinelo S, Hernandez A, et al. Thymic function-related markers within the thymus and peripheral blood: are they comparable? J Clin Immunol 2006;26:96100.

6. Pai SY, Logan BR, Griffith LM, Buckley RH, Parrott RE, Dvorak CC, et al. Transplantation outcomes for severe combined immunodeficiency, 20002009. N Engl J Med 2014;371:434-46.

7. Kwan A, Abraham RS, Currier R, Brower A, Andruszewski K, Abbott JK, et al. Newborn screening for severe combined immunodeficiency in 11 screening programs in the United States. JAMA 2014;312:729-38.

8. Siminovitch KA, Bakhshi A, Goldman P, Korsmeyer SJ. A uniform deleting element mediates the loss of kappa genes in human B cells. Nature 1985;316:260-2.

9. Kanegane H, Futatani T, Wang Y, Nomura K, Shinozaki K, Matsukura $\mathrm{H}$, et al. Clinical and mutational characteristics of X-linked agammaglobulinemia and its carrier identified by flow cytometric assessment combined with genetic analysis. J Allergy Clin Immunol 2001;108:101220.

10. Ballow M. Primary immunodeficiency disorders: antibody deficiency. J Allergy Clin Immunol 2002;109:581-91.

How to cite this article: Rhim JW. Importance of neonatal screening for primary immunodeficiencies. Clin Exp Pediatr 2021;63:519-20. https://doi.org/10.3345/cep.2021.00283 